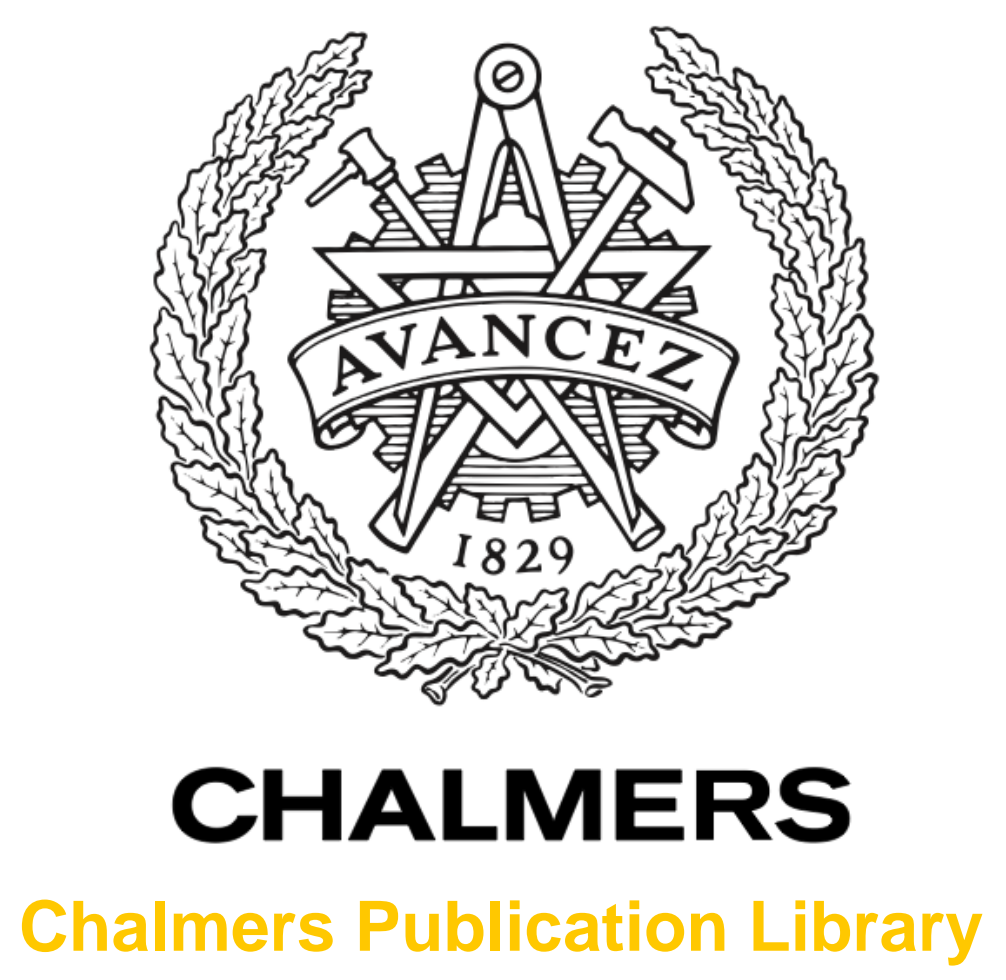

\title{
Performance evaluation of diversity antennas in multipath environments of finite richness
}

This document has been downloaded from Chalmers Publication Library (CPL). It is the author's version of a work that was accepted for publication in:

Proceedings of 6th European Conference on Antennas and Propagation, EuCAP 2012. Prague, 26-30 March 2012

Citation for the published paper:

Jamaly, N. ; Iftikhar, M. ; Rahmat-Samii, Y. (2012) "Performance evaluation of diversity antennas in multipath environments of finite richness". Proceedings of 6th European Conference on Antennas and Propagation, EuCAP 2012. Prague, 26-30 March 2012 pp. 368372.

http://dx.doi.org/10.1109/EuCAP.2012.6205947

Downloaded from: http://publications.lib.chalmers.se/publication/160313

Notice: Changes introduced as a result of publishing processes such as copy-editing and formatting may not be reflected in this document. For a definitive version of this work, please refer to the published source. Please note that access to the published version might require a subscription. 


\section{Performance Evaluation of Diversity Antennas in Multipath Environments of Finite Richness}

\author{
Nima Jamaly \\ Chalmers University of Technology \\ Gothenburg, Sweden 41296 \\ Email: jamaly@chalmers.se
}

\author{
M. Tabish Iftikhar \\ Chalmers University of Technology \\ Gothenburg, Sweden 41296 \\ Email: tabish@student.chalmers.se
}

\author{
Yahya Rahmat-Samii \\ University of California Los Angeles \\ Los Angeles, CA 90095-1594 \\ Email: rahmat@ee.ucla.edu
}

\begin{abstract}
The simulation tool introduced in [1] is extended further to include the emulation of non-uniform multipath environments. This extension creates a trend to generalize the definition of effective diversity gain to be applicable to nonuniform multipath environments too. We introduce the richness threshold as a further metric for diversity performance of multiport antennas. By virtue of this novel parameter, we demonstrate that beam-forming, which is ineffective in a rich isotropic multipath environment, is beneficial in non-rich isotropic multipath environments.
\end{abstract}

\section{INTRODUCTION}

Diversity gain plays a significant role in contemporary wireless communication systems, which are particularly designed to work in multipath environments. The concept of diversity gain is well established in different literatures [2]-[6], and the number of publication addressing it is vast. Yet, from antenna standpoint, there are a few known methods for attaining the maximum realizable apparent or effective diversity gain by a multi-port antenna system associated with certain diversity combining scheme [2] \& [5]. For instance, limited to two-port antennas, the compact formulas provided by [7] presents the quickest approach being restricted to selection combining and maximum ratio combining (MRC) schemes. In contrast, the numerical approach proposed in [8] stands as a general method independent of the number of ports but solely valid for MRC scheme. Nevertheless, both the aforementioned methods are solely credible for rich multipath environments wherein there are numerous incoming waves.

In parallel to those approaches, a different numerical method is introduced in [1], in which they realize samples of the received signals at different ports of a multi-element antenna by emulating an isotropic multipath environment. Having samples of the random received signals enables them to realize all possible diversity schemes, which is advantageous compared to the former methods. Perhaps the main drawback in this approach is associated with computational burden inherent in it. The method has been used for lossless antennas in isotropic environments. The formulas used in the aforementioned simulation tool are further developed in [9] to stand for a general case of lossy antennas.

In addition to being applicable to antennas of arbitrary number of ports, the latter approach benefits from a unique feature. Indeed, one of the variables in this approach is the number of incoming waves in each scenario [1], which is an indicator of richness in the emulated multipath environment. Thus, the current approach makes it possible to study the diversity gain performance of a multi-port antenna in environments of finite richness. This has been performed partially in [10]. Yet, the emulated multipath environments were limited to the isotropic ones. On the contrary, in the current paper, we extend the approach for emulation of non-uniform multipath environments, which is presented in section two. Emulation of multipath environment of non-uniform angle of arrival (AoA) necessitates the proper generalization of the corresponding effective diversity gain's definition, which is the concern of the third section. In section IV, the notion of richness threshold is described with the help of a few simulations on different multi-port antennas. An interesting study is associated with investigation of the richness threshold when using a Butler network. In this effort, a compact formula is presented rendering the total embedded efficiencies of a lossless multi-port antenna in the presence of this microwave network for matchterminations [9, pp.13]. Later, different simulation results are illustrated. These two latter parts are dealt with in section $\mathrm{V}$. Our paper is finalized by a brief conclusion. Note that throughout this paper, we restrict ourselves to MRC diversity scheme and multipath environments of balanced polarization.

\section{Simulation Description}

In this study, simulation has been done by virtue of a software, called multipath environment emulator for performance simulation of radiation terminals (MEST), which is based on the method initially developed in [1]. The embedded element patterns plus scattering parameters of the multi-port antenna are the inputs to MEST. The number of incoming waves as an indicator of richness of environment and the total number of scenarios form its further inputs. MEST outputs different multiple input and multiple output (MIMO) parameters including ergodic capacity as well as different diversity gains. Note that the embedded element patterns along with S-parameters can simply be achieved by virtue of a full-wave software. In this work, the wire structure analysis program (WSAP) as well as computer simulation technology (CST) microwave studio have been used. We need to dedicate some words to briefly describe how MEST conducts the simulation. 
In MEST, the embedded element pattern of antennas are exposed to several random incoming electromagnetic (EM) plane waves. At each realized scenario, there are certain number of incoming EM waves coming from different scatterers located around the terminal. The electric fields of all these plane waves contribute together to give rise to voltages at different ports of our antenna [1] \& [10]. Now, in the second round of realization, the same number of waves are launched coming from other directions which, in turn, create our second voltage samples at the ports of the terminal. This process of realizations continues to eventually result in sufficient number of voltage samples at the input ports of the antenna. By further processing of these voltage samples, we can achieve the corresponding cumulative density function (CDF) curves as well as different MIMO parameters. As a point of caution, note that in parallel with antenna elements, we also need to expose an arbitrary reference antenna to the same set of incoming waves at each scenario and use its received average power for normalization purposes. Throughout this paper, we used a virtual single-port single-polarized isotropic antenna as our reference.

The major point to be elaborated in this paper is to add the emulation of non-uniform AoA. To that purpose, first assume that the coordinate of AoA is denoted by $\Omega(\theta, \psi)$. A uniform distribution of AoA over a sphere necessitates a uniform distribution of $\psi$ on $[0,2 \pi]$ and a sinusoidal distribution of $\theta$ on $[0, \pi][11$, prob. 4.6.1]. Yet, it can be shown that for a non-uniform probability density function (PDF) of AoA in the elevation plane, the distribution of $\theta$ is given by

$$
\mathrm{PDF}_{\theta}=\mathrm{C} \sin (\theta) f(\theta)
$$

where $\mathrm{C}$ is determined to satisfy the general property of a PDF function to integrate to unity, $f(\theta)$ is the truncated version of the corresponding model e.g., Gaussian, double exponential, Laplacian etc., and $\theta \in[0, \pi][12]$. A similar formula can be applied to $\mathrm{PDF}_{\psi}$, except that the sinus function in (1) should be dropped. Now that the PDFs of the coordinates of AoA are known, the question is how to sample from these distributions. In the first step, Marsaglia's Ziggurat algorithm available in MATLAB can be used to realize uniform random samples. If the desired function, $f$, is an invertible function, the inverse transform technique presents an efficient method [11, sec 4-11]. In cases where more complex probability mass functions like Gaussian, Laplacian etc. are of concern, the rejection method is highly recommended [11, pp 123].

\section{General Definition of EfFective Diversity GAIN}

Bear in mind that apparent diversity gain (ADG) is a measure to show how much utilizing multi-element antennas is beneficial in comparison with using only the best single-branch of them. Obviously, it does not contain information about how much well the multi-element antenna under test performs relative to an ideal reference antenna. To address the latter concern, effective diversity gain (EDG) has been coined as an absolute measure of diversity gain [13]. Initially, more for its practical appeal, EDG has been defined solely for isotropic environments and based on a single port reference antenna. However, by virtue of the simulation approach elaborated in the frame of this paper, we are now in a position to generalize the definition of EDG for any arbitrary unbalanced polarized non-uniform environment. Therefore, we redefine EDG as the ratio between the strengths of the diversity signal and that of the isotropic dual-polarized, dual-port ideal reference antenna in $1 \%$ level of their CDFs, measured or simulated in ideally similar multipath environments. Thus, the general definition of EDG can be recast as

$$
\mathrm{EDG}=\mathrm{MEG}_{\max } \cdot \mathrm{ADG}
$$

in which $M E G_{\max }$ denotes the mean effective gain of the best branch. For a particular case of multipath environment of balanced polarization, EDG associated to isotropic single-port (previous definition) and dual-port (new definition) reference antennas can be related through the expressions given in Table I. Note that based on the given definition, EDG is not always necessarily less than ADG.

\section{Richness Threshold: A New Performance METRIC}

Let us presume the best multipath circumstance, wherein incoming waves of different AoAs as well as incoming waves of different polarizations are independent. The preliminary results shown in [10] illustrate that in this multipath environment the ultimate diversity performance of a multi-port antenna system cannot be realized unless there are sufficient number of incoming waves. Indeed, the distributions of the random received signals in all ports do not converge to Rayleigh unless the number of incoming waves exceeds a certain threshold. Dependent on the shapes of the embedded patterns of the elements as well as the properties of the AoAs, this threshold called richness threshold is a single unique number rendering an overall information about performance of a multi-port antenna system.

It has been reported that in a typical multipath environment the number of incoming waves at a receiving multi-element antenna system is five or six [14]-[16]. A number of 20 incoming waves in a rich scattering environment seems also presumably acceptable. However, as our preliminary simulations show [10], for a vast group of multi-element antennas with an average amount of mean effective directivity this number of incoming waves does not result in the optimum performance. This fact reveals the importance of threshold richness as a critical performance metric in addition to correlations and embedded element efficiencies (or MEGs).

\section{A. Simulation Results}

As an initial example, let us study the influence of number of incoming waves on the diversity gain performance of four equidistant quarter wavelength monopoles above a perfect electric conductor plane (PEC). A schematic of this fourport antenna is shown in Fig.1 where the distance between the elements is $d=0.5 \lambda_{\circ}$, in which $\lambda_{\circ}=0.3 \mathrm{~m}$, near 
TABLE I

EDG VERSUS ADG, STRICTLY ASSOCIATED TO A RICH MULTIPATH ENVIRONMENT OF BALANCED POLARIZATION.

\begin{tabular}{|c|c|c|}
\hline Reference Antenna & Uniform AoA & Non-uniform AoA \\
\hline Isotropic single port & $\mathrm{EDG}=e_{\text {tot }_{\max }} \cdot \mathrm{ADG}$ & $\mathrm{EDG}=2 \mathrm{MEG}_{\max } \cdot \mathrm{ADG}$ \\
\hline Isotropic dual port & $\mathrm{EDG}=\frac{1}{2} e_{\text {tot }_{\max }} \cdot \mathrm{ADG}$ & $\mathrm{EDG}=\mathrm{MEG}_{\max } \cdot \mathrm{ADG}$ \\
\hline
\end{tabular}

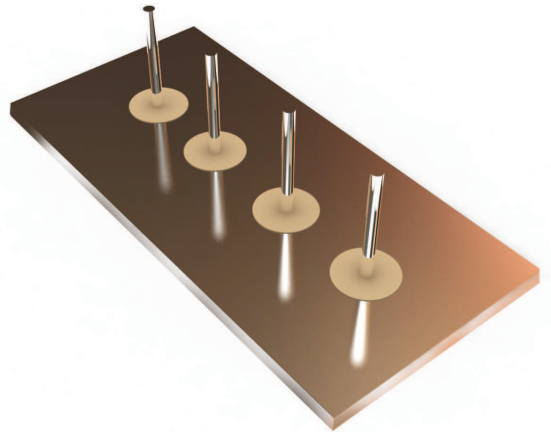

Fig. 1. Four equidistant quarter wavelength collinear monopoles above a PEC plane. The distance between the elements is $d=0.5 \lambda_{\circ}$.

the resonance frequency of the isolated elements. The $50 \Omega$ embedded far field functions of this multi-port antenna system have been achieved by WSAP which is based on the method of moments. Concerning the total embedded efficiencies, it can be shown that in case of a lossless structure, they are given by the diagonal elements of

$$
e_{\text {tot }}=\operatorname{diag}\left(\mathbf{I}-\mathbf{S}_{a}^{\dagger} \mathbf{S}_{a}\right)
$$

where $\mathbf{I}$ is an $n \times n$ unitary matrix, $\mathbf{S}_{a}$ is the input scattering parameters of the $n$-port antenna, and $\cdot^{\dagger}$ stands for Hermitian transpose. Now that the necessary parameters are known, MEST can used to realize the EDG versus the number of the incoming waves at each scenario. The number of scenarios in these simulations exceeds $10^{6}$ rendering a negligible relative error in EDG. The results of simulations are illustrated in Fig.2. According to this figure, it is clear that the antenna under test in an isotropic multipath environment requires at least 28 independent incoming waves in order to give its best performance. To show how threshold richness as well as EDG depend on the AoA distribution, we study a different case. A configuration of four horizontal dipoles above a PEC plane is shown in Fig.3. The embedded patterns are achieved by use of CST. The simulation of non-uniform AoA in MEST is associated with truncated Gaussian distribution in elevation plane, whose specification is shown in the legend, and uniform distribution in the azimuth plane. The results are illustrated in Fig.4. It is clear that not only the ultimate EDG, but also the threshold richness is also different.

\section{Performance at the Presence of A Butler NETWORK}

In the current section, we show by simulation that how the richness threshold can be improved by using an ideal Butler

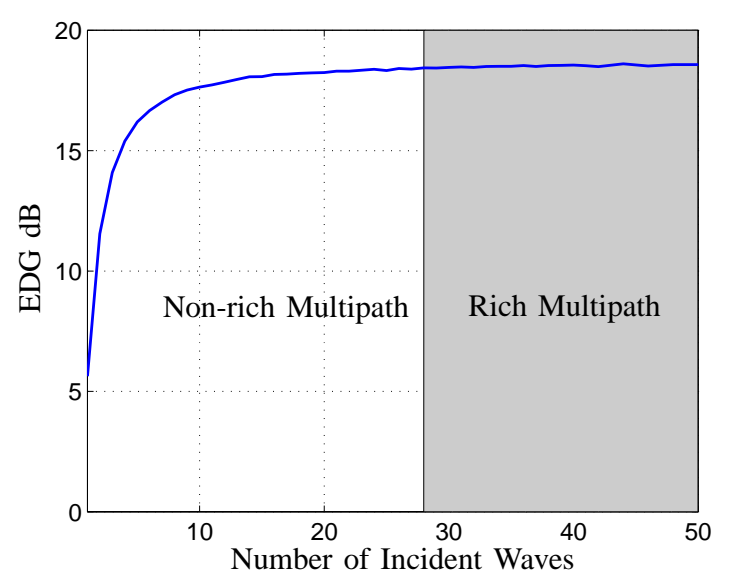

Fig. 2. EDG versus the number of incoming waves for four monopoles above a PEC plane in an isotropic environment. The simulation frequency is $f=1050 \mathrm{MHz}$.

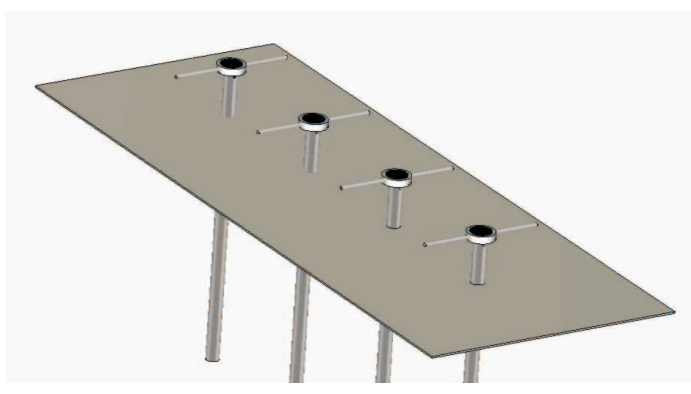

Fig. 3. Four equidistant horizontal dipoles above a PEC plane. The effects of dual split balun is also included in our simulation.

network. An ideal Butler network is a lossless passive reciprocal network. It transforms -for instance- the signal at one of its input ports to the corresponding ones at all its output ports with constant amplitude but certain phase progression among them. At the absence of coupling between the elements, this excitation scheme leads to different directive beams oriented towards different directions in space. For simplicity, let us refer to the ports at the input of the Butler network as beam ports.

In order to achieve the embedded patterns as well as the embedded efficiencies at the beam ports, we use the Sparameters of the Butler network given in [17]. The embedded patterns can be obtained by applying the associated excitation schemes at the ports of the antenna, e.g., element ports. At the presence of a transparent Butler network (i.e., it is matched at both input and output sides) terminated in match impedances, 


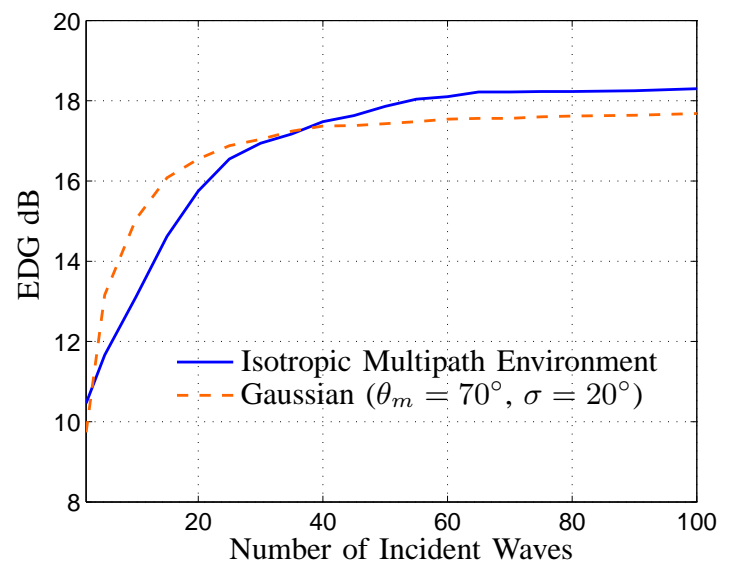

Fig. 4. EDG versus the number of incoming waves for four horizontal dipoles above a PEC plane in isotropic and non-uniform multipath environments. The simulation frequency is around resonance.

the total embedded efficiencies at the beam ports are given by

$$
e_{\text {beam }}=\operatorname{diag}\left(\mathbf{I}-\boldsymbol{\Gamma}^{\dagger} \boldsymbol{\Gamma}\right)
$$

where

$$
\boldsymbol{\Gamma}=\mathbf{T} \mathbf{S}_{a} \mathbf{T}^{\mathrm{T}}
$$

in which ${ }^{\mathrm{T}}$ stands for transpose operator, and $\mathbf{T}$ (for a fourport Butler network) is provided in [17]. For particular case of $d=0.5 \lambda_{\circ}$ distance between the four monopoles above a PEC plane, the total embedded efficiencies versus frequencies at beam ports and element ports are shown in Fig.5. The corresponding correlations are also shown in Fig.6. Note that at $f=1050 \mathrm{MHz}$ the total efficiencies are similar and the corresponding correlations are pretty negligible. On the other hand, similar to simulations conducted in the previous section, we can use MEST to achieve the EDG at the beam ports, whose results are presented in Fig.7. It is clear that despite presenting negligible correlation and similar total embedded efficiencies, the threshold richness associated with each case is considerably different. Indeed, the presence of a Butler network has decreased the latter metric from 28 to less than 6 number of incoming waves. Please note that the two cases give the same EDGs in a rich scattering environment. Yet, in non-rich scattering environment, the EDG at beam ports is far better.

An identical simulation can be carried out for four horizontal dipoles above a PEC plane with $d=0.5 \lambda_{\circ}$. Our simulations demonstrate again similar embedded efficiencies and negligible correlations around the resonance point. The simulation results for this example is given in Fig. 8 which is associated to an isotropic multipath environment. Although it is not shown, the results illustrate that the threshold richness related to the element ports exceeds 150 whereas it is 65 at the beam ports. The ultimate diversity gain for both cases is $\mathrm{EDG}=18.6 \mathrm{~dB}$.

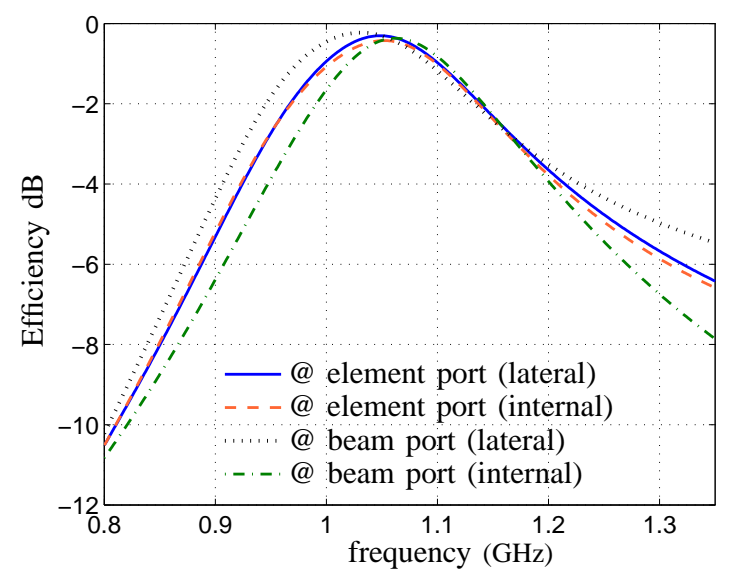

Fig. 5. Total embedded efficiencies at element ports and beam ports for four monopoles above a PEC plane $\left(d=0.5 \lambda_{\circ}\right)$. The two lateral and internal elements have similar efficiencies.

\section{CONCLUSION}

In this paper, we have detailed a simple way for emulation of non-uniform environments by sampling from a given probability distribution for the AoAs. A compact formula is presented for the total embedded element efficiencies at the element ports as well as the beam ports. In addition to correlation and total embedded element efficiencies (or in general MEGs), a further performance metric has been introduced, called richness threshold. The threshold richness is a single performance metric, which is the number of independent random incoming waves required resulting in converged Rayleigh distribution of random signal at all ports of a multiport antenna system. The lower this performance metric is, the better the overall performance of the multi-port antenna in multipath environments. On the contrary, by a numerical simulation, we showed that at the absence of coupling between different elements, the presence of a Butler network does not affect the total embedded efficiencies and correlations around the resonance point. Therefore, the ultimate achievable diversity gain does not change by Butler network. But, this network improves the threshold richness considerably, which is rewarding.

\section{REFERENCES}

[1] K. Rosengren and P.-S. Kildal, "Radiation efficiency, correlation, diversity gain and capacity of six monopole antenna array for a MIMO system: Theory, simulation and measurement in reverberation chamber," Proceedings IEE, Microwaves Antennas and Propagation, vol. 152, no. 1, pp. 7-16, 2005, see also Erratum published in August 2006.

[2] W. C. Jakes, Microwave Mobile Communications. John Wiley \& Sons, 1974.

[3] R. Vaughan and J. Andersen, "Antenna diversity in mobile communications," IEEE Transactions on Vehicular Technology, vol. 36, no. 4, pp. 149-172, November 1987.

[4] R. G. Vaughan and J. B. Andersen, Channels, Propagation and Antennas for Mobile Communication. Instituation for Electrical Engineers, IET, 2003.

[5] A. Goldsmith, Wireless Communications. Cambridge University Press, 2005.

[6] P.-S. Kildal and C. Orlenius, Chapter 58 in Antenna Engineering Handbook, 4th ed., J. L. Volakis, Ed. McGraw-Hill, 2007. 


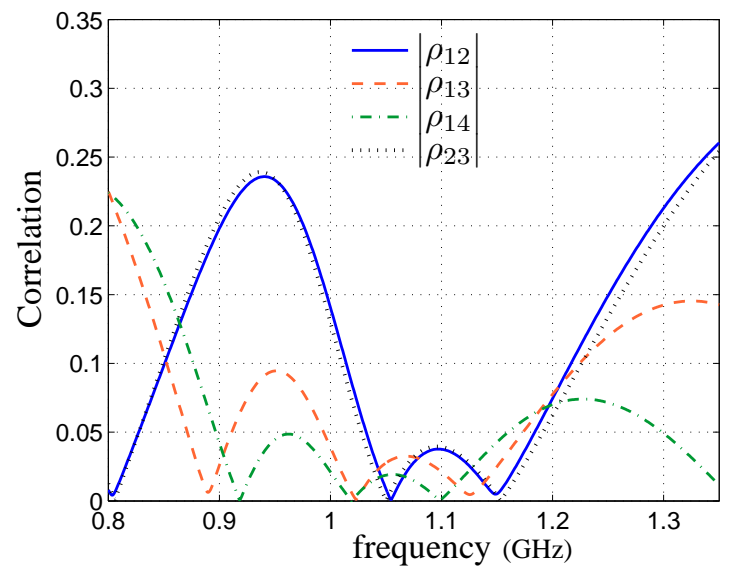

(a) Correlation at element ports.

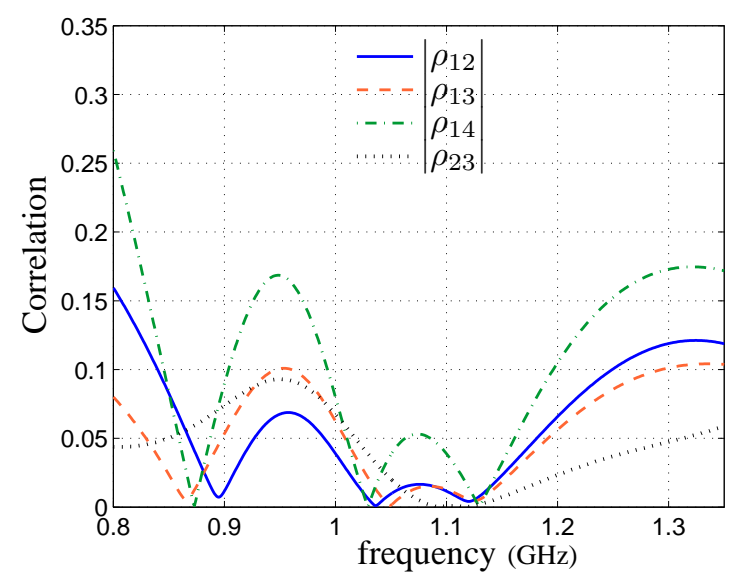

(b) Correlation at beam ports.

Fig. 6. Absolute value of complex correlation versus frequency at element ports and beam ports for four monopoles above a PEC plane $\left(d=0.5 \lambda_{\circ}\right)$.

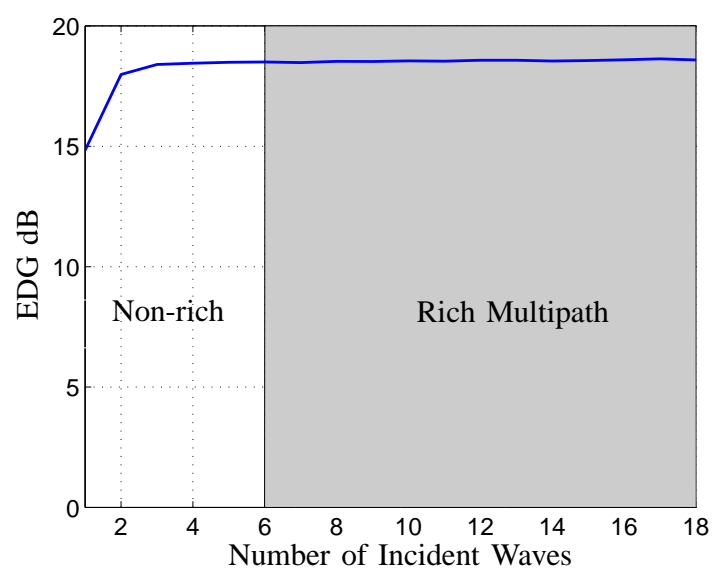

Fig. 7. EDG at beam ports versus the number of incoming waves for four monopoles above a PEC plane. The simulation frequency is $f=1050 \mathrm{MHz}$.

[7] N. Jamaly, P.-S. Kildal, and J. Carlsson, "Compact formulas for diversity gain of two-port antennas," IEEE Antennas and Wireless Propagation

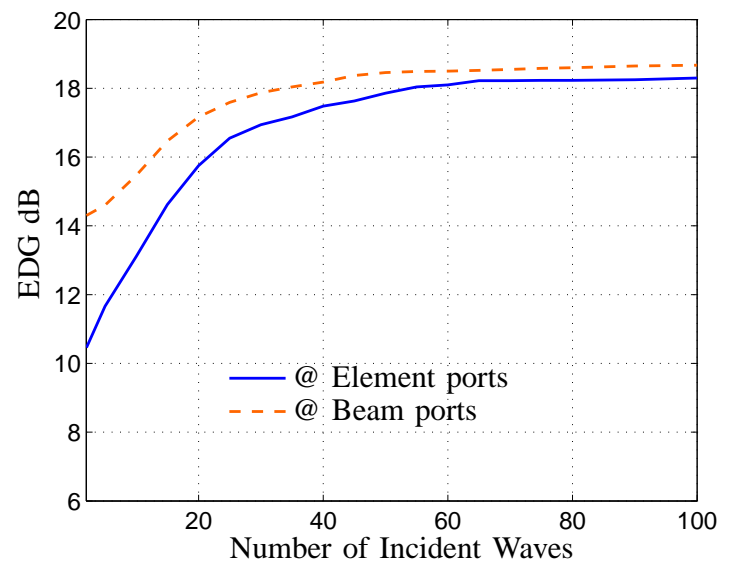

Fig. 8. EDG versus the number of incoming waves for four horizontal dipoles above a PEC plane in an isotropic environment. The simulation frequency is around the resonance point.

Letters, vol. 9, pp. $970-973,2010$.

[8] O. Norklit, P. Teal, and R. Vaughan, "Measurement and evaluation of multi-antenna handsets in indoor mobile communication," IEEE Transactions on Antennas and Propagation, vol. 49, no. 3, pp. 429437, March 2001.

[9] N. Jamaly, "Spatial characterization of multi-element antennas," Chalmers University of Technology, available online at http://publications.lib.chalmers.se/records/fulltext/137635.pdf, Tech. Rep., 2011.

[10] N. Jamaly, H. Zhu, P.-S. Kildal, and J. Carlsson, "Performance of directive multi-element antennas versus multi-beam arrays in MIMO communication systems," in 2010 Proceedings of the Fourth European Conference on Antennas and Propagation (EuCAP), April 2010, pp. $1-5$.

[11] G. Grimmett and D. Stirzaker, Probability and Random Processes, 3rd ed. Oxford, 2001.

[12] K. Kalliola, K. Sulonen, H. Laitinen, O. Kiveks, J. Krogerus, and P. Vainikainen, "Angular power distribution and mean effective gain of mobile antenna in different propagation environments," IEEE Transactions on Vehicular Technology, vol. 51, no. 5, pp. 823-838, 2002.

[13] P.-S. Kildal, K. Rosengren, J. Byun, and J. Lee, "Definition of effective diversity gain and how to measure it in a reverberation chamber," Microwave and Optical Technology Letters, vol. 34, no. 1, pp. 56-59, July 2002.

[14] F. Ikegami and S. Yoshida, "Analysis of multipath propagation structure in urban mobile radio environments," IEEE Transactions on Antennas and Propagation, vol. 28, no. 4, pp. 531-537, 1977.

[15] S. Sakagami, "Experimental results on multipath propagation in 900 $\mathrm{MHz}$ mobile communication path-amplitude-frequency characteristics and angle of arrival," IEICE Japan Transactions, vol. J70-B, no. 12, pp. 1522-1528, 1987.

[16] T. Taga, "Analysis for mean effective gain of mobile antennas in land mobile radio environments," IEEE Transactions on Vehicular Technology, vol. 39, no. 2, pp. 117-131, 1990.

[17] G. P. Riblet, "Simplified 8-port S-matrix of the general reciprocal lossless matched 4-way divider with output port isolation," IEEE Microwave and Guided Wave Letters, vol. 5, no. 11, 1995. 